\title{
LONG MEMORY IN FINANCIAL MARKETS: A HETEROGENEOUS AGENT MODEL PERSPECTIVE
}

\author{
MIN ZHENG ${ }^{\dagger}$, RUIPENG LIU ${ }^{\ddagger}$, AND YOUWEI LI ${ }^{\S, \aleph ~}$ \\ ${ }^{\dagger}$ China Institute for Actuarial Science, Central University of Finance and Economics, Beijing 100081, China \\ $\ddagger$ Department of Finance, Business School, Deakin University, Melbourne, Australia

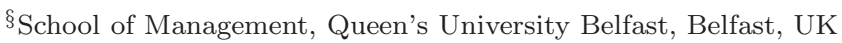 \\ ${ }^{\aleph}$ Hull University Business School, University of Hull, Hull, UK
}

\begin{abstract}
During last decades, studies on asset pricing models witnessed a paradigm shift from rational expectation and representative agent to an alternative, behavioral view, where agents are heterogeneous and boundedly rational. In this paper, we model the financial market as an interaction of two types of boundedly rational investors - fundamentalists and chartists. We examine the dynamics of the market price and market behavior, which depend on investors' behavior and the interaction of the two types of investors. Numerical simulations of the corresponding stochastic model demonstrate that the model is able to replicate the stylized facts of financial time series, in particular the long-term dependence (long memory) of asset return volatilities. We further investigate the source of the long memory according to asset pricing mechanism of our model, and provide evidences of long memory by applying the modified $R / S$ analysis. Our results demonstrate that the key parameter that has impact on the long memory is the speed of the price adjustment of the market maker at the equilibrium of demand and supply.
\end{abstract}

Keywords: Heterogeneity, bounded rationality, asymmetrical beliefs, long memory, modified $R / S$ test

Date: Latest version: February 28, 2018.

Acknowledgements: Financial supports from the National Natural Science Foundation of China (No. 71571197) and Beijing Natural Science Foundation (No. 9152016) are also gratefully acknowledged.

$¥$ Corresponding author: ruipeng.liu@deakin.edu.au. 


\section{INTRODUCTION}

The explanatory power of heterogeneous agent models (HAMs) in finance to various stylized facts and anomalies in financial markets has been evidenced by rapidly growing literature. With different groups of traders having different expectations about future prices, asset price endogenously fluctuates. For instance, by considering two types of traders, typically fundamentalists and chartists, Beja and Goldman (1980), Day and Huang (1990), Chiarella (1992) and Lux (1995) were amongst the first to have shown that interaction of agents with heterogeneous expectations may lead to market instability. HAMs have successfully explained various types of market behavior, such as the long-term swing of market prices from the fundamental price, asset bubbles and market crashes, showing a potential to characterize and explain the stylized facts (LeBaron, 2006 and Gaunersdorfer and Hommes, 2007) and various power law behavior (Alfarano et al., 2005 and $\mathrm{He}$ and Li, 2008) observed in financial markets ${ }^{1}$.

Aa a salient stylized facts, long memory in volatility has been extensively studied. This paper extends the literature examining the source of long memory. We introduce a parsimonious asset pricing model with boundedly rational agents including fundamentalists, chartists using percentage retracement strategies, and a market maker adjusting market price proportional to previous price in a nonlinear fashion. We find that price limitation mechanism of the market maker is essential to control the fluctuation amplitude of the market price, and the speed of the price adjustment of the market maker can be a source of the volatility persistence.

In related HAMs literature on the explanation of long memory in volatility, explanatory mechanisms have been proposed based on the underlying deterministic dynamics, i.e., He and Li (2007, 2015, 2017), Gaunersdorfer et al. (2008) and He, Li and Wang $(2016)^{2}$. The first mechanism is based on the local stability and Hopf bifurcation, explored in He and Li (2007). Essentially, on the parameter space of the deterministic model, near the Hopf bifurcation boundary, the fundamental price can be locally stable but globally unstable. Due to the nature of Hopf bifurcation, such instability leads to periodic oscillations around the fundamental price. Then triggered by fundamental and market noises, He and Li (2007) show that the interaction of fundamentalists and trend followers chasing risk-adjusted trends and the interplay of the noises and the underlying deterministic dynamics can be the source of power-law behavior in return volatility. The estimated models with and without

\footnotetext{
${ }^{1}$ Early comprehensive survey papers include: Hommes (2006), LeBaron (2006), Chiarella et al. (2009), Lux (2009b), Chen et al. (2012), He (2014) and Westerhoff and Franke (2014). For the latest survey, see Dieci and He (2018).

${ }^{2}$ Other mechanisms are also discussed in Lux and Alfarano (2016) including stochastic herding (Alfarano et al. 2005), and stochastic demand (Franke and Westerhoff, 2011, 2012).
} 
switching share the same underlying deterministic mechanism. However, economically, they provide different behavioral mechanisms. In He and Li (2015), a constant market fraction model is used to examine the potential mechanism. The estimated parameters show that, with the dominance of trend followers (about 60\%), the model is able to match closely the power-law behavior of the DAX30. With both switching and no-switching investors, the estimated model in He and Li (2017) shows that the market is dominated by these traders (about 70\%) who consistently switch between two strategies. It is traders' adaptive behavior that generates the power-law behavior. The second mechanism proposed in Gaunersdorfer et al. (2008) is characterized by the coexistence of two locally stable attractors with different size. The interaction of the deterministic dynamics and noise processes can then trigger the switching between the two attractors and endogenously generate volatility clustering and long memory in volatility. Dieci et al. (2006) show that the extended market fraction model with switching investors can display such co-existence of locally stable fundamental price and periodic cycle. More recently, He et al. (2016) provide the conditions on the coexistence of a locally stable steady state and a locally stable invariant circle of the underlying nonlinear deterministic model.

In this paper, we introduce a parsimonious asset pricing model with boundedly rational agents who are specified as using simple heuristics in their decision making in the market maker scenario. The difference from the existing literature is that the price adjustment by the market maker is a proportion based on the previous prices rather than an absolute amount. Within this price adjustment mechanism, the level of the fundamentals can affect the whole system. The impact of the bounded rationality of investors on the volatility of a risky asset becomes complex, which triggers the intrinsic characteristics of market volatility. The long-term dependence of asset return volatilities has been ubiquitous in the financial markets. We identify the sources of the long memory according to asset pricing mechanism of our model. For the empirical testing of long memory, there exist various methods in the literature. It is well-known that the classical $R / S$ test is weak and Lo's rescaled $R / S$ test is stringent (see, for instance, Lillo and Farmer, 2004). We adopt Lo's modified $R / S$ test for long-memory in our study.

The framework of the paper is following. Section 2 introduces the model and discusses characteristics of the corresponding deterministic system. Section 3 considers the stochastic model and the impacts of model parameters on the market price. Section 4 presents results of Lo's modified $R / S$ test. Some conclusions are stated in Section 5. 


\section{MODEL}

In building of HAMs, one of the key aspects is the expectation feedback mechanism: agents' decisions are based upon predictions of endogenous variables whose actual values are determined by the behavior of agents (Brock and Hommes, 1998). Within this framework, adaptation, evolution, heterogeneity, and even learning can be incorporated (Hommes, 2001; Chiarella and He, 2002, 2003; Chiarella et al., 2002, 2006 and Zheng et al., 2017). The promising perspectives of HAMs have been testified by many empirical studies of $\mathrm{HAMs}^{3}$.

Following on HAMs literature, we consider a market with one risky asset (e.g. stock, index or fund) and one risk free asset. Let $P_{t}$ denote the price (ex dividend) per share of the risky asset at time $t$, and let $y_{t}$ be the stochastic dividend process of the risky asset. The risk free asset is perfectly elastically supplied at gross return $R \geq 1^{4}$. At the same time, the market consists of different types of heterogeneous traders whose excess demands are based on different trading rules. In this paper, we consider two types of heterogeneous traders ${ }^{5}$, that is fundamental analysts (fundamentalists) and technical analysts (chartists).

Fundamentalists - In the market, fundamentalists (denoted by agent $f$ ) know the fundamental information of the risky asset, such as the fundamental price. So the decision of the fundamentalists is depended upon the fundamental information and further their excess demand $D_{t}^{f}$ is based on the spread between the actual price $P_{t}$ and the fundamental price $F_{t}$, which can be expressed as

$$
D_{t}^{f}=\alpha\left(F_{t}-P_{t}\right)
$$

where $\alpha>0$ is the reaction coefficient of the fundamentalists, which denotes the slope of the downward-sloping demand function and represents the mean-reverting belief of the fundamentalists. When $F_{t}>P_{t}$, the fundamentalists believe that the

\footnotetext{
${ }^{3}$ They include commodity markets (Baak, 1999 and Chavas, 2000), stock markets (Boswijk et al., 2007; Franke, 2009; Franke and Westerhoff, 2011, 2012; Chiarella et al., 2012, 2014; Schmitt and Westerhoff, 2014 and He and Li, 2015, 2017), foreign exchange markets (Westerhoff and Reitz, 2003; Manzan and Westerhoff, 2005; De Jong et al., 2010; Dieci and Westerhoff, 2010 and ter Ellen et al., 2013), housing market (Dieci and Westerhoff, 2016 and Zheng et al., 2017), mutual funds (Goldbaum and Mizrach, 2008), option markets (Frijns et al., 2010), oil markets (ter Ellen and Zwinkels, 2010), and CDS markets (Chiarella et al., 2015). Also, HAMs have been estimated with contagious interpersonal communication by Gilli and Winker (2003), Alfarano et al. (2005), Lux (2009a, 2012), and other works reviewed in Li et al. (2010) and Chen et al. (2012).

${ }^{4}$ Usually, the gross return is $R=1+r / K$ where $r$ stands for a constant risk-free rate per annual and $K$ stands for the frequency of trading period per year. Typically, $K=1,12,52$ and 250 for trading period of year, month, week and day, respectively.

${ }^{5}$ The model can be extended to three or more agents.
} 
risky asset is undervalued and so they like to hold a long position. Contrarily, the price is regarded as overvaluation and so they hold a short position.

Chartists - Chartists (denoted by agent c) don't know the fundamental information and they believe that the charting signals from the past prices can discover some information about the spot price of the risky asset. So they prefer the cheaper thumb strategies, such as simple moving average, percentage retracement, correlation analysis, and other popular technical methods in financial market. In our paper, we mainly analyze the effect of percentage retracement (PR) because it is seldom studied in the previous HAMs literature. A retracement is a countertrend move. Retracements are based on the thought that prices will reverse or "retrace" a portion of the previous movement before resuming their underlying trend in the original direction. For example, 33\%, 50\% and 66\% retracement rates are well-known as illustrated in Figure 1. In particular, when the chartists believe $50 \%$ retracement, then if the short down-adjustment of the market during the rise trend of the price is not across the line of $50 \% \mathrm{PR}$, the chartists think that the down-trend hasn't made, the market is just in a temporary adjustment and it will go up after the adjustment; otherwise the down-adjustment crosses $50 \% \mathrm{PR}$, then they think that the down-trend of the price is doomed and the rise move is over. In addition, some chartists draw similarities between $33 \%, 50 \%$, and $66 \%$ and the Fibonacci numbers of $38.2 \%, 50 \%$, and $61.8 \%$, this is Fibonacci retracement.

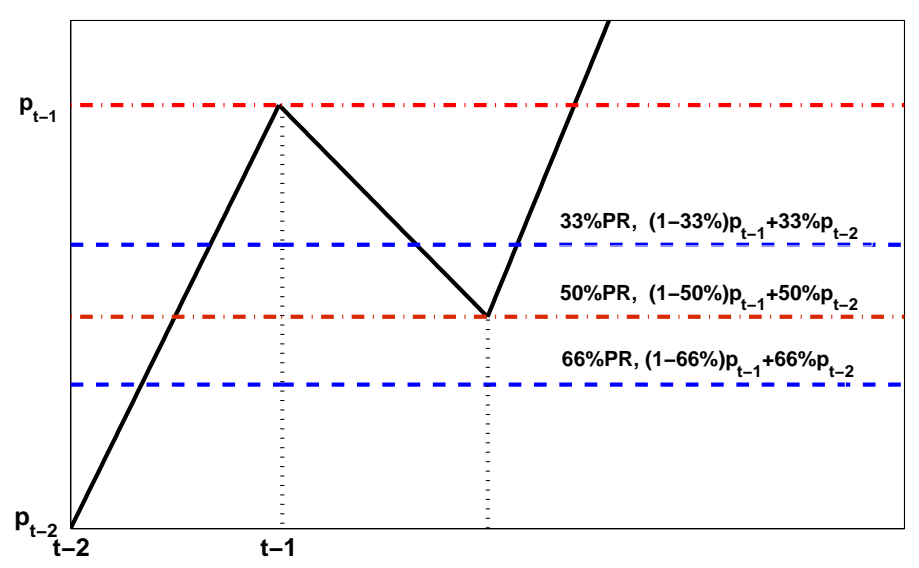

Figure 1. Percentage Retracement (PR)

Therefore, the reference price of the percentage retracement strategy adopted by the chartists can be expressed by the weighted average of historical prices, that is

$$
\widehat{P}_{t}=(1-\omega) P_{t-1}+\omega P_{t-2},
$$


which is called the expectation price of the chartists and where $\omega \in[0,1]$ represents the coefficient of the retracement strategy. In particular, if $\omega=0$, then the expectation of the chartists to the price is the previous price, that is $\widehat{P}_{t}=P_{t-1}$, which is so-called naive expectation. If $\omega=1$, then $\widehat{P}_{t}=P_{t-2}$, which means that the chartists regard the lag-2 price as their expectation price. If $\omega=0.5, \widehat{P}_{t}$ is the simple moving average of length 2 . Thus, a trading signal to the chartists is defined as the difference $\left(\delta_{t}\right)$ between the current price $P_{t}$ and the expectation price $\widehat{P}_{t}$, that is $\delta_{t}=P_{t}-\widehat{P}_{t}$. When $\delta_{t}>0$, the chartists believe it indicates that the price is in the increasing trend and then they want to hold a long position; otherwise, they like to take a short position. By mathematical expression, the excess demand $\left(D_{t}^{c}\right)$ of the chartists can be written as

$$
D_{t}^{c}=g\left(\delta_{t}\right),
$$

where $g(\delta)$ is a nonlinear smooth function of the price difference $\delta$. In the financial market, technical analysts have different actions to different information and they are cautious for the big rise or fall of the market price. So $g$ should have the following general properties ${ }^{6}: \exists \delta^{*}<0, \delta^{* *}>0$ such that

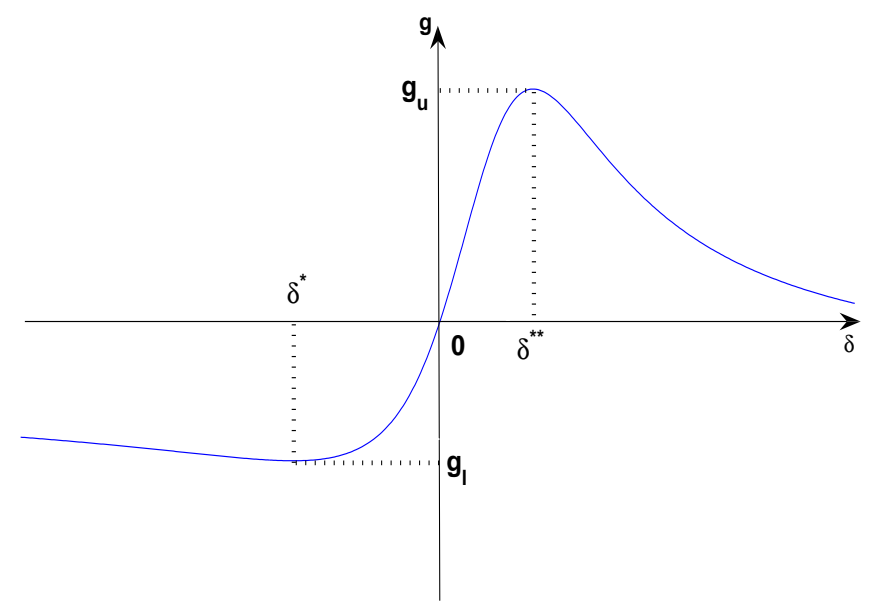

FiguRE 2. $g$-function

$$
\begin{gathered}
g(0)=0, \delta g(\delta)>0 \text { for } \delta \neq 0 \\
g^{\prime}(\delta)>0 \text { for } \delta^{*}<\delta<\delta^{* *}, \quad g^{\prime}(\delta)<0 \text { for } \delta<\delta^{*} \text { or } \delta>\delta^{* *}
\end{gathered}
$$

\footnotetext{
${ }^{6}$ Usually $g$ is assumed to be an $S$-shape function, e.g. $g(\delta)=\mathbb{u} \tanh (\mathbb{v} \delta)(u>0, \mathbb{v}>0)$. Note that under the assumption, $g(\cdot)$ is symmetric and $\lim _{\delta \rightarrow \pm \infty}|g(\delta)|=\mathbb{u}<\infty$, which means that the actions of the chartists to the positive and negative trading signals are completely equally reverse operations and the chartists are cautious but not very cautious when the price difference $\delta$ is large (either positive or negative).
} 


$$
\lim _{\delta \rightarrow \delta^{*}} g(\delta)=g_{l}<+\infty, \quad \lim _{\delta \rightarrow \delta^{* *}} g(\delta)=g_{u}>-\infty .
$$

In particular, in this paper, we take $g(\delta)=\frac{a \delta}{1+b \delta+c^{2} \delta^{2}}$ where $a>0, c>0, b \in$ $(-2 c, 2 c)$ and then $g$ satisfies the characteristics in (2.1) and other features as follows:

$$
\begin{aligned}
g(0) & =0, \quad \delta g(\delta)>0 \text { for } \delta \neq 0, \\
g_{u} & \equiv g_{\max }=g\left(\frac{1}{c}\right)=\frac{a}{2 c+b} \\
g_{l} & \equiv g_{\min }=g\left(-\frac{1}{c}\right)=-\frac{a}{2 c-b}
\end{aligned}
$$

These mean that when the price has no trend (that is $\delta_{t}=0$ ), the chartists do nothing and only wait and see. But when there is positive (negative) trend, they will increase a long (short) position and the position is limited because of the wealth constraint.

$$
g^{\prime}(\delta)>0 \text { for }|\delta|<\frac{1}{c} \text { and } g^{\prime}(\delta)<0 \text { for }|\delta|>\frac{1}{c} .
$$

The parameter $c$ measures the confident level of the chartists to extrapolate. For $|\delta|<\frac{1}{c}$, the chartists' demand increases as the price difference $\delta$ increases. However, for $|\delta|>\frac{1}{c}$, the chartists' demand decreases as $\delta$ increases. The maximum demand amount achieves when $|\delta|=\frac{1}{c}$. In other words, the chartists are confident for their strategies when the changes in $|\delta|$ are up to the level of $\frac{1}{c}$. However, the chartists become less confident and cautious for their strategies when the changes in $|\delta|$ excess the level of $\frac{1}{c}$. In particular, as $c \rightarrow 0$, the chartists' demand tends to increase for all $\delta$ while when $c$ is far away from zero, the chartists' demand increases sharply as $\delta$ are small and then decreases.

(3) $\lim _{\delta \rightarrow \pm \infty} g(\delta)=0$ which means that when the price difference $\delta$ between the current price and the expectation price of the chartists is becoming large, the chartists become cautious and reduce their demand.

(4) The parameter $a=g^{\prime}(0)>0$ measures the chartists' extrapolation when $\delta$ is small. For small (large) values of $a$, the chartists' demand is insensitive (sensitive) to small values of $\delta$, which may characterize unwillingness (willingness) of the chartists to get into the market when the real price is deviated from its expectation price.

(5) The parameter $b$ measures the symmetry of the chartists' response to the changes of $\delta$. For $b=0$, the chartists' long and short positions are symmetric with respect to the changes of $\delta$. However, for $b \neq 0$, the position of the chartists for positive and negative information is not symmetric, which represents that the chartists have no equal actions toward good news and bad news. The phenomenon is called the asymmetric effect. In particular, for $b<0$, the chartists believe a bull market by increasing (decreasing) their 
maximum long (short) position when $\delta$ is positive (negative) and for $b>0$, the chartists believe a bear market by decreasing (increasing) their maximum long (short) position when $\delta$ is positive (negative). We call $b$ the bull-bear coefficient, in brief B-coefficient.

Price adjustment mechanism - In line with Beja and Goldman (1980), Chiarella, He and Hommes (2006), we assume that the risky asset price is set period by period via a market maker mechanism upon the aggregate excess demand $D_{t}$ which is given by

$$
D_{t}=n_{f, t} D_{t}^{f}+n_{c, t} D_{t}^{c}
$$

where $n_{q, t}(q \in\{f, c\})$ is the market fraction of agent $q$ at time $t$ and $n_{f, t}+n_{c, t}=1$. But it is necessary to point out that the market maker mechanism in the previous studies updates the price based on the absolute amount of the price, which cannot avoid the case of the negative price. In addition, the difference of price is independent of fundamental price, which is not realizable. Here we adopt the mechanism to adjust the price in the relative instead of absolute amount. That is to say,

$$
P_{t+1}=P_{t}\left(1+\mathcal{S}\left(D_{t}\right)\right)
$$

where $\mathcal{S}(d)$ is a nonlinear smooth function of the excess demand $d$, satisfying

$$
\begin{gathered}
\mathcal{S}(0)=0, \mathcal{S}^{\prime}(d)>0, \lim _{d \rightarrow \pm \infty} \mathcal{S}^{\prime}(d)=0, \\
\lim _{d \rightarrow+\infty} \mathcal{S}(d)=S_{u}<+\infty, \lim _{d \rightarrow-\infty} \mathcal{S}(d)=S_{l} \geq-1 .
\end{gathered}
$$

Thus, the relative amount of the price adjustment is bounded, which is consistent with the fact that the price has the boundary. Denote $\mu=\mathcal{S}^{\prime}(0)$ which measures the speed of price adjustment of the market maker to the excess demand at the equilibrium of demand and supply. When $\mu$ is small, the market maker adjusts cautiously the price change at the equilibrium of demand and supply.

In this paper, we take $\mathcal{S}(d)=k \tanh (\gamma d)$ where $0<k<1$ and $\gamma>0$. Then the price adjustment speed of the market maker $\mu$ is determined by the adjustment amplitude $k$ and the reaction speed $\gamma$, that is $\mu=k \gamma$. Therefore, if the market maker wants to cautiously adjust the price change, then he needs to decrease his price adjustment speed $\mu$ (that is to decrease his price adjustment amplitude and/or reaction speed).

Fitness Measure and Fraction Evolution - At time $t$, agent $q$ has the fitness measure $\pi_{q, t}(q=f, c)$ as their realized net profit:

$$
\pi_{q, t}=\left(P_{t}+y_{t}-R P_{t-1}\right) D_{t-1}^{q}-C_{q}
$$


where $C_{q} \geq 0$ is the cost of agent $q$. Since fundamental information is expensive, the fundamentalists should pay no less than the chartists to obtain information. So we assume that $C=C_{f}-C_{c} \geq 0$.

More generally, one can introduce additional memory into the performance measure $U_{q, t}$, by considering a weighted average of net realized profit, as follows:

$$
U_{q, t}=\pi_{q, t}+\eta U_{q, t-1},
$$

where the parameter $\eta \in[0,1)$ represents the memory of the cumulated fitness function.

Each agent has survival risk. To avoid the risk, all agents prefer a better strategy in order to gain more profit. Hence, similarly to Brock and Hommes (1998) and Chiarella, He and Hommes (2006), the market fraction $n_{q, t}$ of agent $q(q=f, c)$ is given by the discrete choice probability

$$
n_{q, t}=\frac{e^{\beta U_{q, t}}}{N_{t}}
$$

where $N_{t}=e^{\beta U_{f, t}}+e^{\beta U_{c, t}}$ and the parameter $\beta(\geq 0)$ measures the sensitivity of performance, that is how fast different type agents in the market switch each other. In particular, as $\beta=\infty$, the total fraction is held by the investors choosing the optimal strategy in each period whilst as $\beta=0$, the fundamentalists and chartists take the even market fractions at all times. See Brock and Hommes (1997, 1998) for a more extensive discussion of the discrete choice setup for predictor selection.

Summarizing the above analysis, we can get the following dynamical system:

$$
\left\{\begin{aligned}
P_{t+1} & =P_{t}\left[1+\mathcal{S}\left(n_{f, t} D_{t}^{f}+n_{c, t} D_{t}^{c}\right)\right], \\
n_{f, t} & =\frac{e^{\beta U_{f, t}}}{e^{\beta U_{f, t}}+e^{\beta U_{c, t}}}, \quad n_{c, t}=1-n_{f, t}, \\
U_{q, t} & =\left(P_{t}+y_{t}-R P_{t-1}\right) D_{t-1}^{q}-C_{q}+\eta U_{q, t-1}, q \in\{f, c\}, \\
D_{t}^{f} & =\alpha\left(F_{t}-P_{t}\right), \quad D_{t}^{c}=g\left(P_{t}-(1-\omega) P_{t-1}-\omega P_{t-2}\right) .
\end{aligned}\right.
$$

Let $U_{t}=U_{f, t}-U_{c, t}=\left(\pi_{f, t}-\pi_{c, t}\right)-C+\eta U_{t-1}$. Then system (2.6) can be rewritten into

$$
\left\{\begin{array}{l}
P_{t+1}=P_{t}\left[1+\mathcal{S}\left(\frac{e^{\beta U_{t}}}{e^{\beta U_{t}}+1} \alpha\left(F_{t}-P_{t}\right)+\frac{1}{e^{\beta U_{t}}+1} g\left(P_{t}-(1-\omega) P_{t-1}-\omega P_{t-2}\right)\right)\right] \\
U_{t+1}=\left(P_{t+1}+y_{t+1}-R P_{t}\right)\left[\alpha\left(F_{t}-P_{t}\right)-g\left(P_{t}-(1-\omega) P_{t-1}-\omega P_{t-2}\right)\right]-C+\eta U_{t} .
\end{array}\right.
$$

When we assume that the fundamental price and dividend both follow constant processes, that is $F_{t} \equiv F^{*}>0$ and $y_{t}=F_{t}(R-1) \equiv F^{*}(R-1)$ denoted by $\bar{y}$. Then system $(2.7)$ can be written into the following deterministic 4-dimensional dynamical 
system $^{7}$ :

$$
\left\{\begin{array}{l}
P_{t+1}=P_{t}\left[1+\mathcal{S}\left(\frac{e^{\beta U_{t}}}{e^{\beta U_{t}}+1} \alpha\left(F^{*}-P_{t}\right)+\frac{1}{e^{\beta U_{t}}+1} g\left(P_{t}-(1-\omega) P_{t-1}-\omega P_{t-2}\right)\right)\right] \\
U_{t+1}=\left(P_{t+1}+\bar{y}-R P_{t}\right)\left[\alpha\left(F^{*}-P_{t}\right)-g\left(P_{t}-(1-\omega) P_{t-1}-\omega P_{t-2}\right)\right]-C+\eta U_{t}
\end{array}\right.
$$

The dynamics of the deterministic model (2.8) is analyzed in Zheng et al. (2009), and the properties of (2.8) are as follows.

Proposition 2.1. For the deterministic dynamical system (2.8), assume $\eta \in[0,1)$. Denote $U^{*}=-\frac{C}{1-\eta}, n_{f}^{*}=\frac{e^{\beta U^{*}}}{e^{\beta U^{*}}+1}, n_{c}^{*}=1-n_{f}^{*}, \bar{\alpha}:=F^{*} \mu n_{f}^{*} \alpha$ and $\bar{a}:=F^{*} \mu n_{c}^{*} a$. Then

(1) there always exist two steady states $\left(P_{1}^{*}, U_{1}^{*}\right)=\left(0, \frac{\alpha F^{*} \bar{y}-C}{1-\eta}\right)$, and $\left(P_{2}^{*}, U_{2}^{*}\right)=$ $\left(F^{*}, U^{*}\right)$.

(2) $\left(P_{1}^{*}, U_{1}^{*}\right)$ is unstable for all parameters $(\bar{a}, \bar{\alpha}, \omega)$.

(3) $\left(P_{2}^{*}, U_{2}^{*}\right)$ is locally asymptotically stable $(L A S)$ for $(\bar{a}, \bar{\alpha}, \omega) \in D$, where

$$
D=\left\{(\bar{a}, \bar{\alpha}, \omega): 0<\omega \leq 1,0<\bar{a}<\frac{1}{\omega}, \max \left\{0, \frac{(\omega \bar{a}+1)((1+\omega) \bar{a}-1)}{\bar{a} \omega}\right\}<\bar{\alpha}<2+2 \bar{a}(1-\omega) ;\right.
$$

$$
\omega=0,0<\bar{a}<1,0<\bar{\alpha}<2+2 \bar{a}\} .
$$

(4) At $\bar{\alpha}=2+2 \bar{a}(1-\omega),\left(P_{2}^{*}, U_{2}^{*}\right)$ undergoes a flip bifurcation.

(5) At $\bar{\alpha} \bar{a} \omega=\bar{a}+\bar{a}^{2}\left(\omega+\omega^{2}\right)-1,\left(P_{2}^{*}, U_{2}^{*}\right)$ can have one pair of complex conjugate eigenvalues on the unit cycle and in addition,

(i) when $(1+\omega) \bar{a}+1-\bar{\alpha} \neq 2 \cos (2 \pi / q)(q=2, \ldots, 6)$, a (generalized) Neimark-Sacker bifurcation occurs;

(ii) when $0<\omega<\frac{1}{2(1-\cos (2 \pi / q))}(q=2,3, \ldots), 1$ : q-resonance occurs at $(\bar{a}, \bar{\alpha})=\left(\frac{1}{1-\omega+2 \omega \cos (2 \pi / q)}, \frac{2(1-\cos (2 \pi / q))(2 \omega \cos (2 \pi / q)+1)}{1-\omega+2 \omega \cos (2 \pi / q)}\right) ;$

(iii) when $\omega=0,1$ : q-resonance $(q=2,3,4)$ occurs at $(\bar{a}, \bar{\alpha})=(1,2-$ $2 \cos (2 \pi / q))$.

\footnotetext{
${ }^{7}$ Note, taking $\log$ in $(2.8)$, the $\log$ price dynamic is given by

$$
p_{t+1}=p_{t}+\$\left(D_{t}\right)
$$

where $p_{t}=\log P_{t}$ and $\$(\cdot)=\log (1+\mathcal{S}(\cdot))$. System $(*)$ seems consistent with the absolute amount of price adjustment, but in fact, if the price follows the absolute adjustment rule, that is,

$$
P_{t+1}=P_{t}+\mathcal{S}\left(D_{t}\right)
$$

then the fundamental value will not affect the dynamic by a transformation $x_{t}=P_{t}-F^{*}$. However, in $(*)$, the fundamental value always exists explicitly and plays the important role in the evolution of prices.
} 


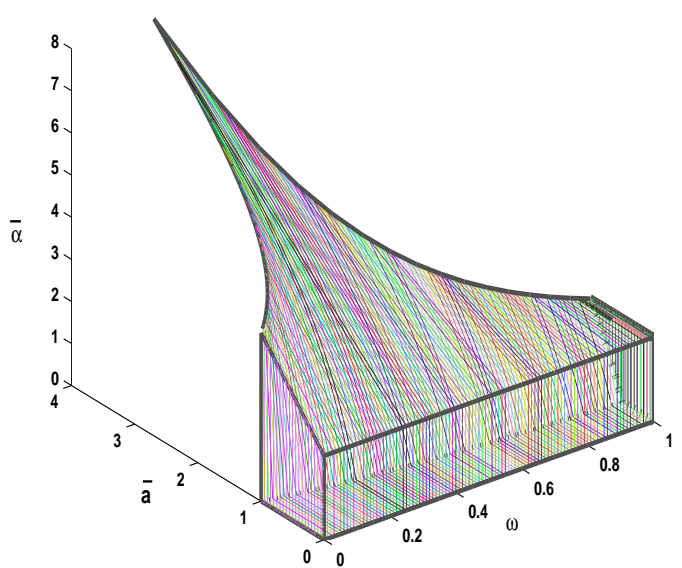

(a)

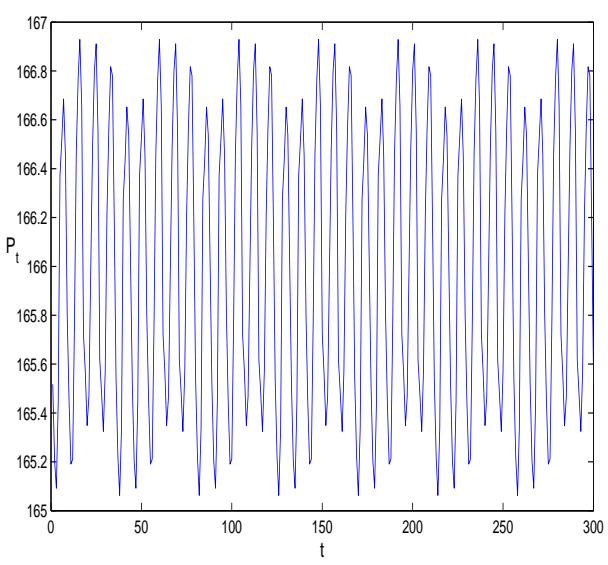

(c)

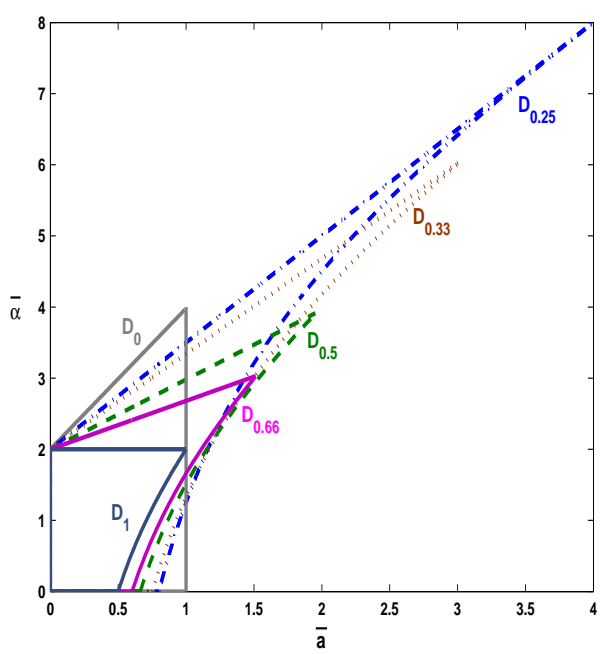

(b)

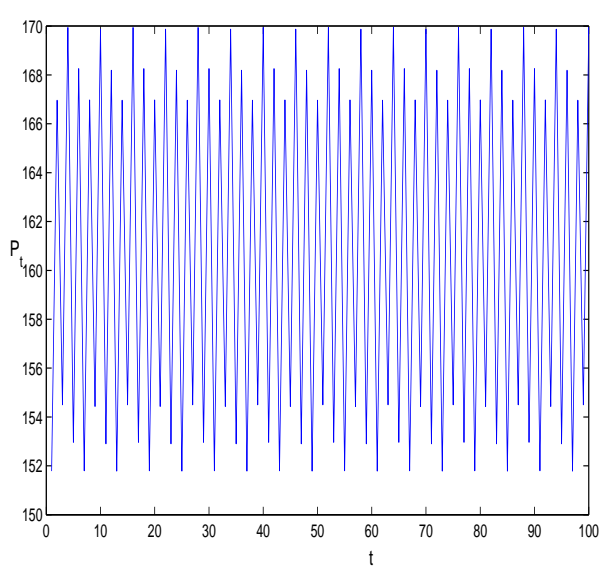

(d)

FiguRE 3. (a) The stable region of the steady state $\left(P_{2}^{*}, U_{2}^{*}\right)$ in the parameter space of $(\bar{a}, \bar{\alpha}, \omega)$. (b) $D_{\omega^{*}}$ is denoted as the projection of $D$ onto the plane $\omega=\omega^{*}$. (c) Time series when the parameters pass the boundary $\bar{\alpha} \bar{a} \omega=\bar{a}+\bar{a}^{2}\left(\omega+\omega^{2}\right)-1$. (d) Time series when the parameters pass the boundary $\bar{\alpha}=2+2 \bar{a}(1-\omega)$.

System (2.8) has two steady states. The price of the first steady state $\left(P_{1}^{*}, U_{1}^{*}\right)$ is zero. For this steady state, it is always unstable. This is because the product with zero price has no value and this type product will die out. This phenomenon is consistent with the real market. For the second steady state $\left(P_{2}^{*}, U_{2}^{*}\right)$, the price is the fundamental value $F^{*}$ of the risky asset and we call $\left(P_{2}^{*}, U_{2}^{*}\right)$ the fundamental steady state. The stability property of the fundamental steady state $\left(P_{2}^{*}, U_{2}^{*}\right)$ depends 
on the parameters $\omega, \bar{\alpha}=F^{*} \mu n_{f}^{*} \alpha$ and $\bar{a}=F^{*} \mu n_{c}^{*} a$. Here the market maker's price adjustment speed $\mu$ and the fundamental price $F^{*}$ both are scaling factors ${ }^{8}$ for $(\bar{a}, \bar{\alpha})$ and can change the stability of the fundamental steady state. Given the scaling factors, $\bar{\alpha}$ and $\bar{a}$ are determined by the fraction weighted (at the fundamental steady state) reaction coefficients of the fundamentalists and chartists, respectively. By Proposition 2.1, we can see if the chartists' retracement strategy $(\omega)$ and the scaled reaction coefficients of the fundamentalists and chartists $(\bar{\alpha}, \bar{a})$ balance each other such that $(\bar{a}, \bar{\alpha}, \omega) \in D$, then the fundamental steady state is stable, as illustrated in Figure $3(\mathrm{a}-\mathrm{b})$. Otherwise, the fundamental steady state will lose its stability and complex phenomena will happen, as shown in Figure 3 (c-d). Therefore, when the heterogeneous beliefs exist in the financial market, they can let the price deviate from and fluctuate around the fundamental information and furthermore, increase the complexity of the whole system. Proposition 2.1 describes the deterministic evolution when there is no noise. If we consider some random factors in the market, then the system could be more complex. In the following sections, we will analyze the properties of system (2.8) with random noises and study its explanatory power to the stylized facts of financial time series.

\section{Stochastic Model}

In Section 2, under the deterministic assumption, we show the impacts of the reaction coefficients $(\alpha, a)$ of the fundamentalists and chartists, the switching speed $(\beta)$ between the two types of investors, the strategy $(\omega)$, confident level $(c)$ and B-coefficient $(b)$ of the chartists, the fundamental price $\left(F^{*}\right)$ and the price adjustment amplitude $(k)$ and reaction speed $(\gamma)$ of the market maker in (2.8). We find that the market price exhibits very rich dynamical characteristics when any of those parameters changes. However, in the real market, the fundamental price usually is not constant but has stochastic fluctuation and moreover, the market is disturbed frequently by the indefinite factors. Therefore, in this section, we will attempt to gain some insights into the different behavior of the corresponding nonlinear stochastic model of (2.8) with randomness arising from noise created by the stochastic fundamental price and/or the market noise.

Stochastic markets repeatedly switch between periods of relative calm and periods of relative turmoil. This feature remains one of the most robust and curious in all of finance. Although much is known about the structure of volatility persistence,

\footnotetext{
${ }^{8}$ In this paper, besides the price adjustment speed of the market maker, the fundamental price is also a scaling factor to affect the stability of the steady state because the market maker adjusts a proportion of the price based on the previous prices. However, in the existing literature, the fundamental price usually does not affect the stability of the steady state because the market maker adjusts the absolute amount of the price.
} 
little is known about its causes. In what follows, the analysis is conducted to explore possible source of volatility persistence. In so doing, we provide some insights into the interplay between deterministic forces and stochastic elements, specially, the potential mechanism in generating realistic time series properties.

In particular, we are interested in the statistical properties of time series, especially in the autocorrelation functions (ACFs) of the returns, and absolute and squared returns. In real financial data, autocorrelation functions of returns are roughly zero at all lags. Autocorrelation functions of volatility measures, such as absolute or squared returns, are positive decaying for all lags. This is the well-known stylized fact known as long memory.

As follows, we want to use a special noise form to probe the stylized fact. Consider the following stochastic dynamical system

$\left\{\begin{array}{l}P_{t+1}=P_{t}\left(1+\mathcal{S}\left(\frac{e^{\beta U_{t}}}{e^{\beta U_{t}+1}} \alpha\left(F_{t}-P_{t}\right)+\frac{1}{e^{\beta U_{t}+1}} g\left(P_{t}-(1-\omega) P_{t-1}-\omega P_{t-2}\right)\right)\right) \cdot m_{t} \\ U_{t+1}=\left(P_{t+1}+\bar{y}-R P_{t}\right)\left[\alpha\left(F_{t}-P_{t}\right)-g\left(P_{t}-(1-\omega) P_{t-1}-\omega P_{t-2}\right)\right]-C+\eta U_{t}\end{array}\right.$

Here the $\log$ fundamental price follows the random walk $\log F_{t+1}=\log F_{t}+\sigma_{F} \varepsilon_{t}$ and $F_{0}=F^{*}$, where $\sigma_{F} \geq 0$ is a constant measuring the volatility of the fundamental return, $\varepsilon_{t}$ follows a standand normal distribution $\mathcal{N}(0,1)$ and $E\left(\log F_{t}\right)=\log F^{*}$. Note that this specification ensures that the log fundamental price changes are stationary. $m_{t}$ is a market noise, which represents the fact that this deterministic model is too simple to capture all dynamics of a financial market. One can interpret this noise term also as coming from noise traders, that is traders whose behavior is not explained by the model but considered as exogenously given. We consider the market noise $m_{t}$ has the form $m_{t}=e^{\sigma_{m} \delta_{t}}$ with $\sigma_{m} \geq 0$ and $\delta_{t}$ follows $\mathcal{N}(0,1)$ which is independent with $\varepsilon_{t}$. If we take returns defined as log price changes, then we can get

$$
r_{t+1}=\log \frac{P_{t+1}}{P_{t}}=r_{t}^{*}+\sigma_{m} \delta_{t},
$$

where $r_{t}^{*}=\log \left(1+\mathcal{S}\left(\frac{e^{\beta U_{t}}}{e^{\beta U_{t}+1}} \alpha\left(F_{t}-P_{t}\right)+\frac{1}{e^{\beta U_{t}+1}} g\left(P_{t}-(1-\omega) P_{t-1}-\omega P_{t-2}\right)\right)\right)$.

In what follows, we take the parameters as follows unless specifically stated:

TABLE 1. Parameter settings

\begin{tabular}{cccccccccccccc}
\hline$\omega$ & $k$ & $\gamma$ & $F^{*}$ & $a$ & $b$ & $c$ & $\alpha$ & $R$ & $\beta$ & $\eta$ & $C$ & $\sigma_{F}$ & $\sigma_{m}$ \\
\hline 0.5 & 0.1 & 0.1 & 166 & 0.966 & 0 & 1 & 1.2 & 1.001 & 1 & 0.2 & 0.1 & 0.03 & 0.00538 \\
\hline
\end{tabular}

From Figure 4, we can see that given the suitable parameters, our model (3.1) can show some characteristics appearing in the real time series. Note that in system (3.1), the deterministic skeleton and random noises interact each other. To clarify the roles of different parameters, first we analyze the dynamic property of the corresponding deterministic system of (3.1) when the parameters vary, and then study 

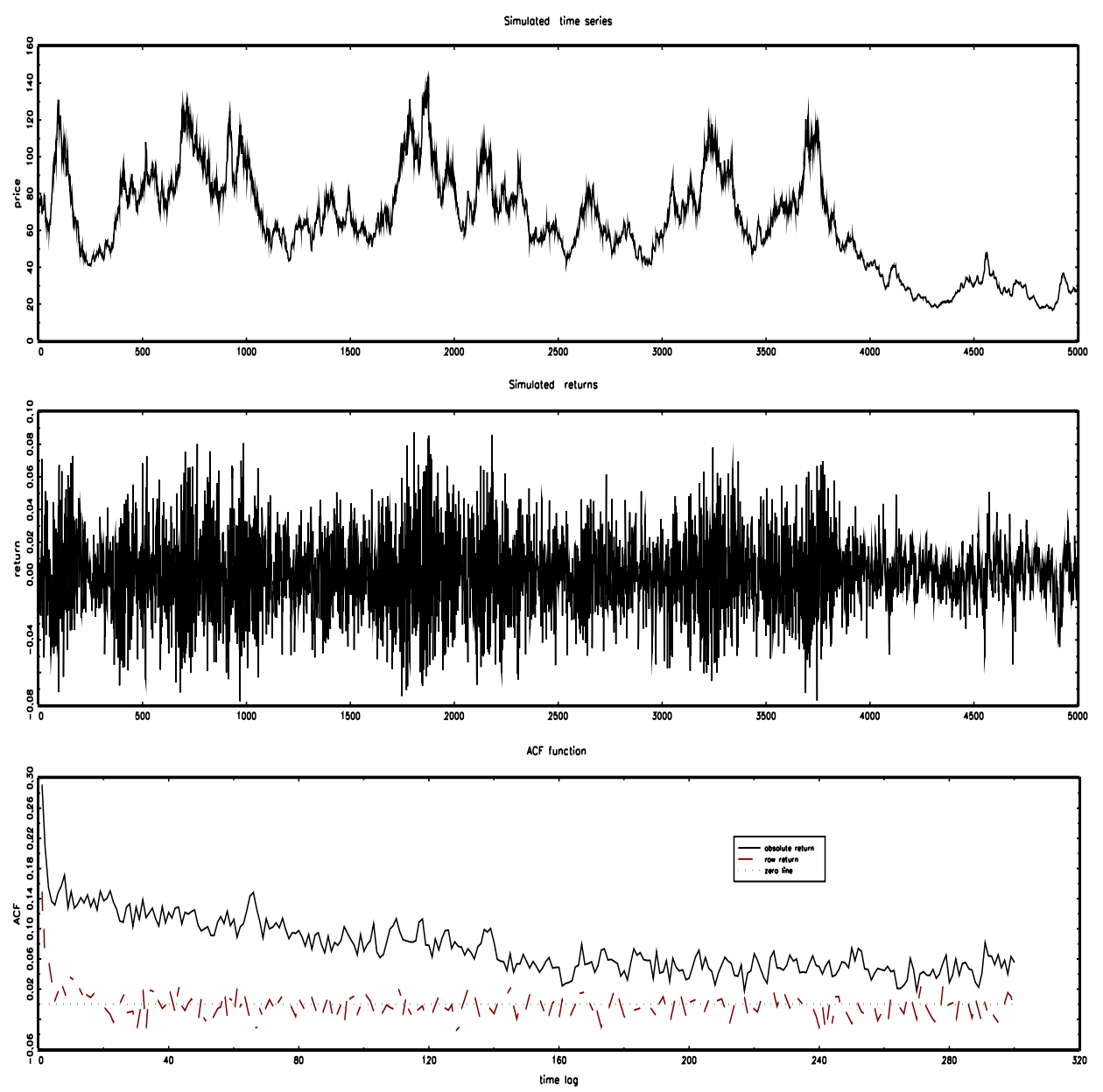

FIgURE 4. Simulation results and the corresponding autocorrelation under the parameters given in Table 1.

the effects of different parameters on the whole system (3.1) in Section 4. In what follows, we respectively analyze the impacts of the parameters ${ }^{9} b, c, k, \gamma, F^{*}, \omega$ and $\beta$.

3.1. The impact of $b$. The parameter $b$ measures the symmetry of the chartists' response to the trend of the price. When $b>0$, it means that the chartists think that the market is bearish so that they prefer selling/buying more/less when a decreasing/increasing price signal appears. Otherwise, when $b<0$, the chartists believe in a bullish market so that an up/down trend of the price triggers a more/less buying/selling demand of the chartists.

We let $b$ change and keep the other parameters as Table 1 . Under the parameters given by Table 1 , for system (2.8), the Neimark-Sacker bifurcation occurs at $a^{*}=$

\footnotetext{
${ }^{9}$ The impact of the fundamentalists and chartists on the system are relative. Here we fix the behavior of the fundamentalists, that is a fixed value of $\alpha$, to analyze the role of the chartists for different parameter values. The impact of fundamentalists' behavior can be similarly analyzed.
} 
0.9671 for any $b$. Especially, at $b=1.4691$, or $-1.4651, P^{*}=F^{*}$ undergoes a Chenciner bifurcation. It means when $-1.4651<b<1.4691$, at $a^{*}=0.9671$, there is a supercritical Neimark-Sacker bifurcation which corresponds to a negative Lyapunov coefficient. Otherwise, there is a subcritical Neimark-Sacker bifurcation which implies two attractors coexist, the stable fundamental steady state $F^{*}$ and a stable limit cycle because of the positive Lyapunov coefficient.

Therefore, similar to He and Li (2007), we take $a=0.966$ near the Neimark-Sacker bifurcation boundary and let $b$ change from -1.6 to 1.6 to analyze the impact of investors' asymmetric belief on the market price in Section 4.

3.2. The impact of $c$. The parameter $c(>0)$ measures the confident level of the chartists. When the price difference between the actual price and the chartists' expectation price is in the range of $[-1 / c, 1 / c]$, the chartists are confident about their strategies so that they like to increase their demand if the price difference increases. Otherwise, if the price difference is beyond $[-1 / c, 1 / c]$, then the chartists are cautious and think that in the market, there exists the possibility of the price reversal so that they prefer decreasing their demand when the price difference increases.

We let $c$ change and keep the other parameters as Table 1. Under the parameters given by Table 1, for system (2.8), the Neimark-Sacker bifurcation occurs at $a^{*}=$ 0.9671 for any $c$. This means $c$ cannot affect the bifurcation point $a^{*}$ while it determines the range of $b$ because of $b \in(-2 c, 2 c)$. Only $b=0$ is always in the range for any $c$.

In Section 4, we still take the value of $a$ near the Neimark-Sacker bifurcation boundary, that is $a=0.966$ as shown in Table 1 and let $c$ change from 0.2 to 1.8 to analyze the impact of $c$.

3.3. The impact of $k$. Note that $\max _{d} \mathcal{S}(d)=k$ and $\min _{d} \mathcal{S}(d)=-k$. In addition, $\mu=k \gamma=\mathcal{S}^{\prime}(0)$ measures the speed of the price adjustment of the market maker at the equilibrium of demand and supply. So the parameter $k$ not only determines the maximum level of the price adjustment but also affects its speed.

We let $k$ change and keep the other parameters as Table 1 . Note that the value of $k$ can change the bifurcation point $a^{*}$ of system (2.8) by Proposition 2.1. To analyze the impact of $k$, we fix the value of $a$ at 0.966 as shown in Table 1 but we should be careful of the bifurcation points of system (2.8) corresponding to different values of $k$ shown in Table 2 and illustrated by Figure 5, which means that under the parameter assumption of Table 1, the fundamental steady state of the corresponding deterministic system of (3.1) is unstable when $k$ exceeds 0.1. In Section 4, we will analyze the impact of increasing $k$ on System (3.1).

3.4. The impact of $\gamma$. Note that $\mu=k \gamma=\mathcal{S}^{\prime}(0)$, so the parameter $\gamma$ affects the speed of the price adjustment of the market maker. 
TABLE 2. Bifurcation values of system (2.8) for different $k$

\begin{tabular}{ccccccccccc}
\hline$k$ & 0.1 & 0.2 & 0.3 & 0.4 & 0.5 & 0.6 & 0.7 & 0.8 & 0.9 & 1.0 \\
\hline$a^{*}$ & 0.9671 & 0.6302 & 0.5490 & 0.5301 & 0.5324 & 0.5419 & 0.5532 & 0.5645 & 0.5748 & 0.5841 \\
\hline
\end{tabular}

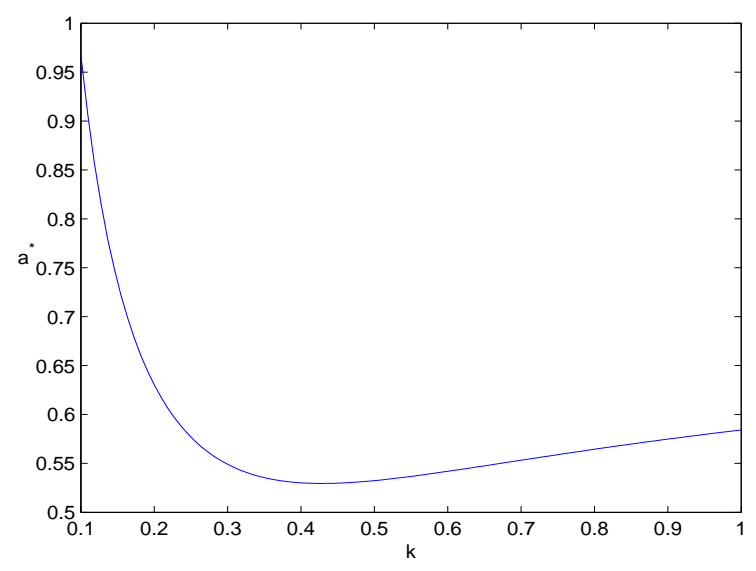

FiguRE 5. For the deterministic system (2.8), the relationship between the bifurcation point $a^{*}$ and the parameter $k$.

We let $\gamma$ change and keep the other parameters as Table 1 . Similarly to $k$, even though we keep $a=0.966$, the bifurcation value of $a^{*}$ of system (2.8) changes with the increase of $\gamma$ shown in Table 3 and illustrated by Figure 6 . In Section 4 , the impact of increasing $\gamma$ on System (3.1) will be analyzed.

TABLE 3. Bifurcation values of system (2.8) for different $\gamma$

\begin{tabular}{ccccccccccc}
\hline$\gamma$ & 0.1 & 0.2 & 0.3 & 0.4 & 0.5 & 0.6 & 0.7 & 0.8 & 0.9 & 1.0 \\
\hline$a^{*}$ & 0.9671 & 0.6302 & 0.5490 & 0.5301 & 0.5324 & 0.5419 & 0.5532 & 0.5645 & 0.5748 & 0.5841 \\
\hline
\end{tabular}

3.5. The impact of $F^{*}$. The parameter $F^{*}$ represents the level of the fundamental price. We let $F^{*}$ change and keep the other parameters as Table 1 . The fundamental value $F^{*}$ is an important factor for the dynamics of the system because it can affect the bifurcation point $a^{*}$ of the corresponding deterministic system (2.8) as shown in Table 4 and illustrated by Figure 7, which means that if we fix $a=0.966$ for the statistical study in Section 4, the fundamental steady state of the corresponding deterministic system of (3.1) changes from stable to unstable when the fundamental value of $F^{*}$ increases from 100 to 200 .

TABLE 4. Bifurcation values of system (2.8) for different $F^{*}$

\begin{tabular}{cccccccccccc}
\hline$F^{*}$ & 100 & 110 & 120 & 130 & 140 & 150 & 160 & 170 & 180 & 190 & 200 \\
\hline$a^{*}$ & 1.4514 & 1.3395 & 1.2466 & 1.1683 & 1.1016 & 1.0441 & 0.9940 & 0.9502 & 0.9115 & 0.8771 & 0.8465 \\
\hline
\end{tabular}




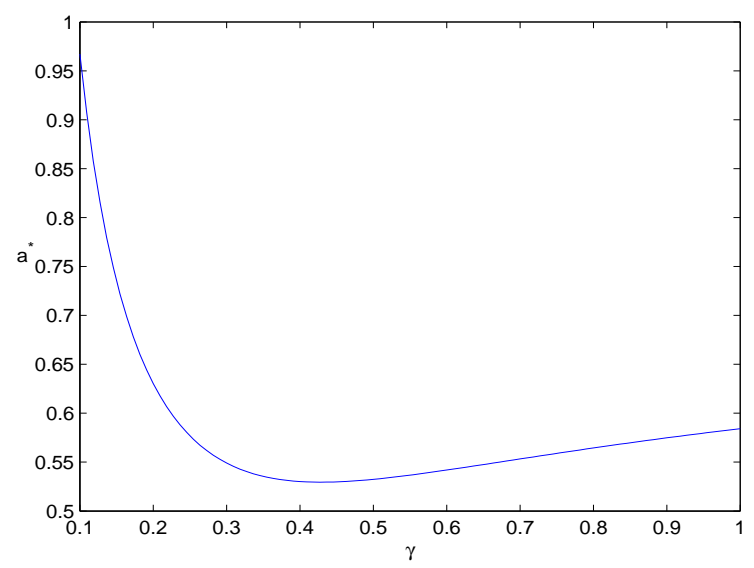

FiguRE 6. For the deterministic system (2.8), the relationship between the bifurcation point $a^{*}$ and the parameter $\gamma$.

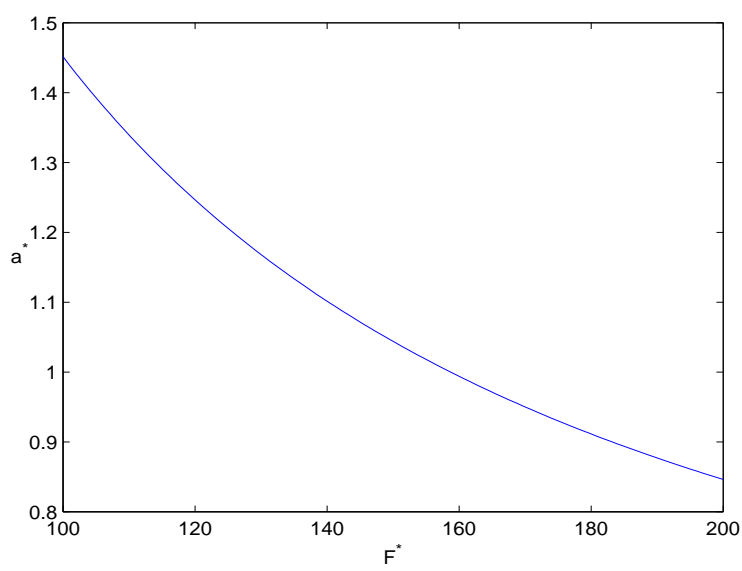

FiguRE 7 . For the deterministic system (2.8), the relationship between the bifurcation point $a^{*}$ and the parameter $F^{*}$.

3.6. The impact of $\omega$. The parameter $\omega \in[0,1]$ represents the coefficient of the retracement strategy, which determines the level of chartists' expectation price. The closer to 0 the parameter $\omega$, the closer to the previous price the expectation price and the closer to 1 the parameter $\omega$, the closer to the lag- 2 price the expectation price.

We let $\omega$ change and keep the other parameters as Table 1. For the corresponding deterministic system (2.8), the relationship between the bifurcation point $a^{*}$ and $\omega$ shown in Table 5 and illustrated by Figure 8. It shows that if we fix $a=0.966$ in Section 4, the fundamental steady state of the corresponding deterministic system of (3.1) changes from stable to unstable when $\omega$ increases from 0 to 1. 
TABLE 5. Bifurcation values of system (2.8) for different $\omega$

\begin{tabular}{cccccccccccc}
\hline$\omega$ & 0 & 0.1 & 0.2 & 0.3 & 0.4 & 0.5 & 0.6 & 0.7 & 0.8 & 0.9 & 1.0 \\
\hline$a^{*}$ & 1.1340 & 1.1173 & 1.0870 & 1.0493 & 1.0085 & 0.9671 & 0.9265 & 0.8876 & 0.8507 & 0.8159 & 0.7833 \\
\hline
\end{tabular}

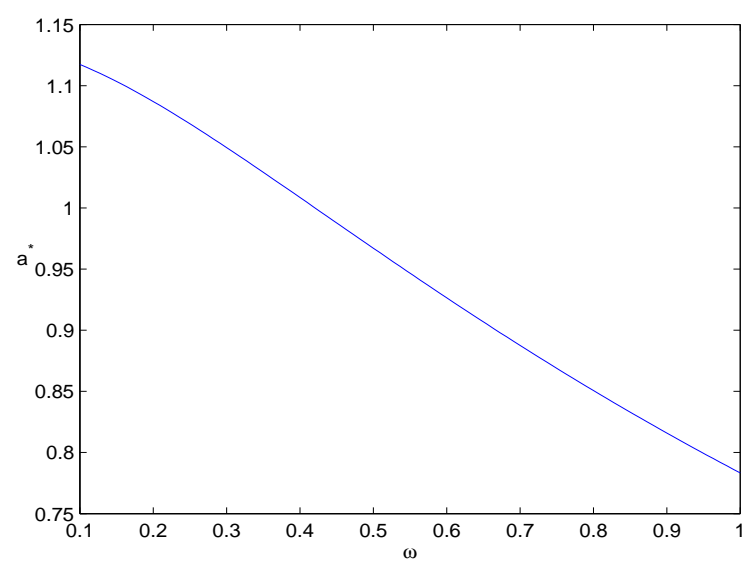

FiguRE 8. For the deterministic system (2.8), the relationship between the bifurcation point $a^{*}$ and the parameter $\omega$.

3.7. The impact of $\beta$. The parameter $\beta$ measures the performance sensitivity. The bigger $\beta$, the faster different type agents switch each other.

We let $\beta$ change and keep the other parameters as Table 1. For the corresponding deterministic system (2.8), the fundamental steady state becomes unstable when $\beta$ exceeds 1 if we fix $a=0.966$, as shown in Table 6 and illustrated by Figure 9. Given the parameter values as shown in Table 1, the impact of increasing $\beta$ on System (3.1) will be analyzed in Section 4.

TABLE 6. Bifurcation values of system (2.8) for different $\beta$

\begin{tabular}{ccccccccccc}
\hline$\beta$ & 1 & 3 & 5 & 7 & 9 & 11 & 13 & 15 & 17 & 19 \\
\hline$a^{*}$ & 0.9671 & 0.8381 & 0.7388 & 0.6622 & 0.6032 & 0.5577 & 0.5226 & 0.4954 & 0.4744 & 0.4582 \\
\hline
\end{tabular}

\section{LO'S MODIFIED $R / S$ ANALYSIS}

The $R / S$ analysis was firstly introduced by Hurst (1951), later refined by Mandelbrot and Wallis (1969), and Mandelbrot and Taqqu (1979). It is based on a heuristic graphical approach. Formally, a time series is divided by equal length of subperiods of $z_{t}(t=1, \ldots, T)$ and the range $R$ of a time series $\left\{z_{t}\right\}_{t=1}^{T}$ is defined as:

$$
R_{T}=\max \sum_{t=1}^{T}\left(z_{t}-\bar{z}\right)-\min \sum_{t=1}^{T}\left(z_{t}-\bar{z}\right)
$$




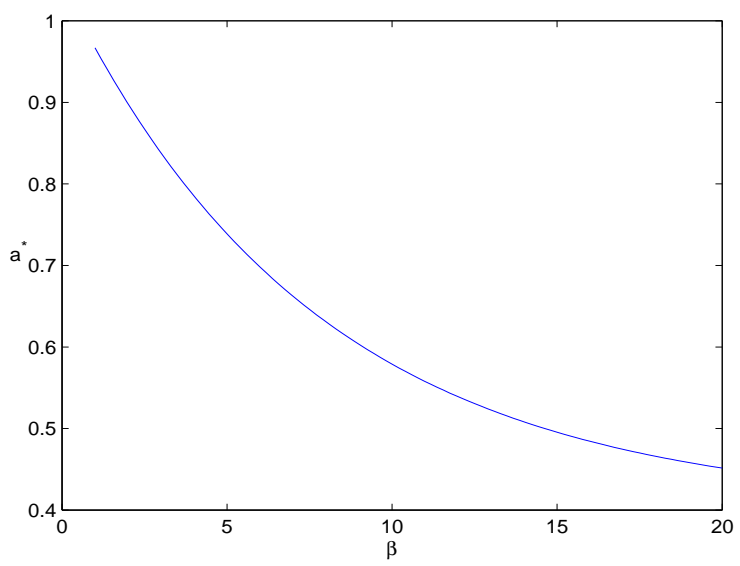

Figure 9. For the deterministic system (2.8), the relationship between the bifurcation point $a^{*}$ and the parameter $\beta$.

Here $\bar{z}$ is the standard estimate of the mean. The range is then rescaled by the sample standard deviation $(S)$, yielding the widely used $R / S$ statistic. Applying Hurst's empirical law with different subperiod sample sizes of $T$ :

$$
\log (R / S)_{T}=C+H \cdot \log (T)
$$

We obtain the Hurst exponent $H$ by running an ordinary least square (OLS) regression. Figure 10 provides an illustration of the Hurst exponent $H$ for the $S \& P 500$ index.

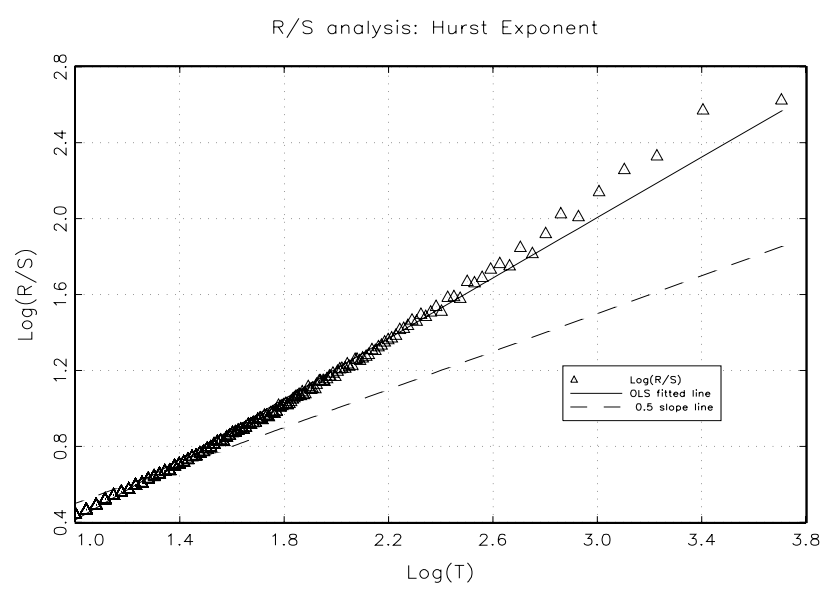

Figure 10. The Hurst exponent of the $S \& P 500$ index based on the $R / S$ analysis.

However, the traditional R/S analysis has two main weaknesses which have been demonstrated by Davies and Harte (1987) and Lo (1991): (1) the distributional properties of the rescaled range are affected by the presence of short-term memory; (2) though there are vast applications of the traditional $R / S$ approach in diverse 
fields, it turned out that no asymptotic distribution theory could be derived for the Hurst exponent $H$ itself. Hence, no explicit hypothesis testing can be performed and the significance of point estimates $H>0.5$ or $H<0.5$ rests on subjective assessment.

In order to obtain robust and convincing results, we adopt the widely used Lo's R/S approach. Lo (1991) proposes a stringent R/S statistic by revising the rescaled range $Q_{\tau}$ ( $\tau$ being the time lag) which adjusts for possible short memory effects by applying the Newey-West heteroscedasticity and autocorrelation consistent estimator in place of the sample standard deviation $S$ with $Q_{\tau}=R_{T} / S_{\tau}$, and

$$
S_{\tau}^{2}=S^{2}+\frac{2}{T} \sum_{j=1}^{\tau} \omega_{j}(\tau)\left\{\sum_{i=j+1}^{T}\left(z_{i}-\bar{z}\right)\left(z_{i-j}-\bar{z}\right)\right\},
$$

with weights defined as

$$
\omega_{j}(\tau)=1-\frac{j}{\tau+1} .
$$

Lo (1991) standardizes the test statistic by introducing a modified $R / S$ statistic $V_{T}$, and derives its explicit distribution function. Under the null hypothesis of no long term memory, the distribution of the random variable $V_{T}=T^{-0.5} Q_{\tau}$ converges weakly to the range of a Brownian bridge. ${ }^{10}$ At the $5 \%$ significance level, the fractiles of the distribution of $V_{T}$ are given as

$$
\lim _{T \rightarrow \infty} \operatorname{Prob}\left\{V_{T} \in[0.809,1.862]\right\}=0.95 .
$$

More critical values of this distribution are tabulated in Lo (1991, Table II).

We firstly conducte the Lo's $R / S$ analysis for empirical stock markets indices, which are the S\&P 500 index (01/07/1996 - 31/08/2016), the Chinese CSI 300 index (08/04/2005 - 28/02/2017), ${ }^{11}$ and the German DAX 30 index (01/07/1996 $22 / 08 / 2016)$. Returns are computed as $\log$ price changes $\mathbb{r}_{t}=\log \left(\mathcal{P}_{t} / \mathcal{P}_{t-1}\right)$, with $\mathcal{P}_{t}$ denoting daily stock indices. Figure 11 presents the empirical plots including the daily level indices and returns. The bottom panel of Figure 11 shows the ACFs of the returns and the absolute values of returns (as proxy of volatilities) for three stock markets. We observe that there are no long memory for return data, but significant hyperbolic decay of the ACF of the absolute values of returns with time lags increasing, which is the typical symbol of long memory in the return volatilities.

The Lo's $R / S$ test results for the empirical data are reported in Table 7 . The left panel reports the Lo's $R / S$ test statistics for different truncation lags $\tau=$ $1,10,50,100,150,250$, respectively. We find that the values are varying with different

\footnotetext{
${ }^{10} \mathrm{Lo}(1991)$ provides the detailed asymptotic distribution and proof.

${ }^{11}$ The CSI 300 index is compiled by the China Securities Index Company, Ltd. The index is normalized relative to a base of 1000 on $31 / 12 / 2004$ and has been calculated and published since 08/04/2005.
} 

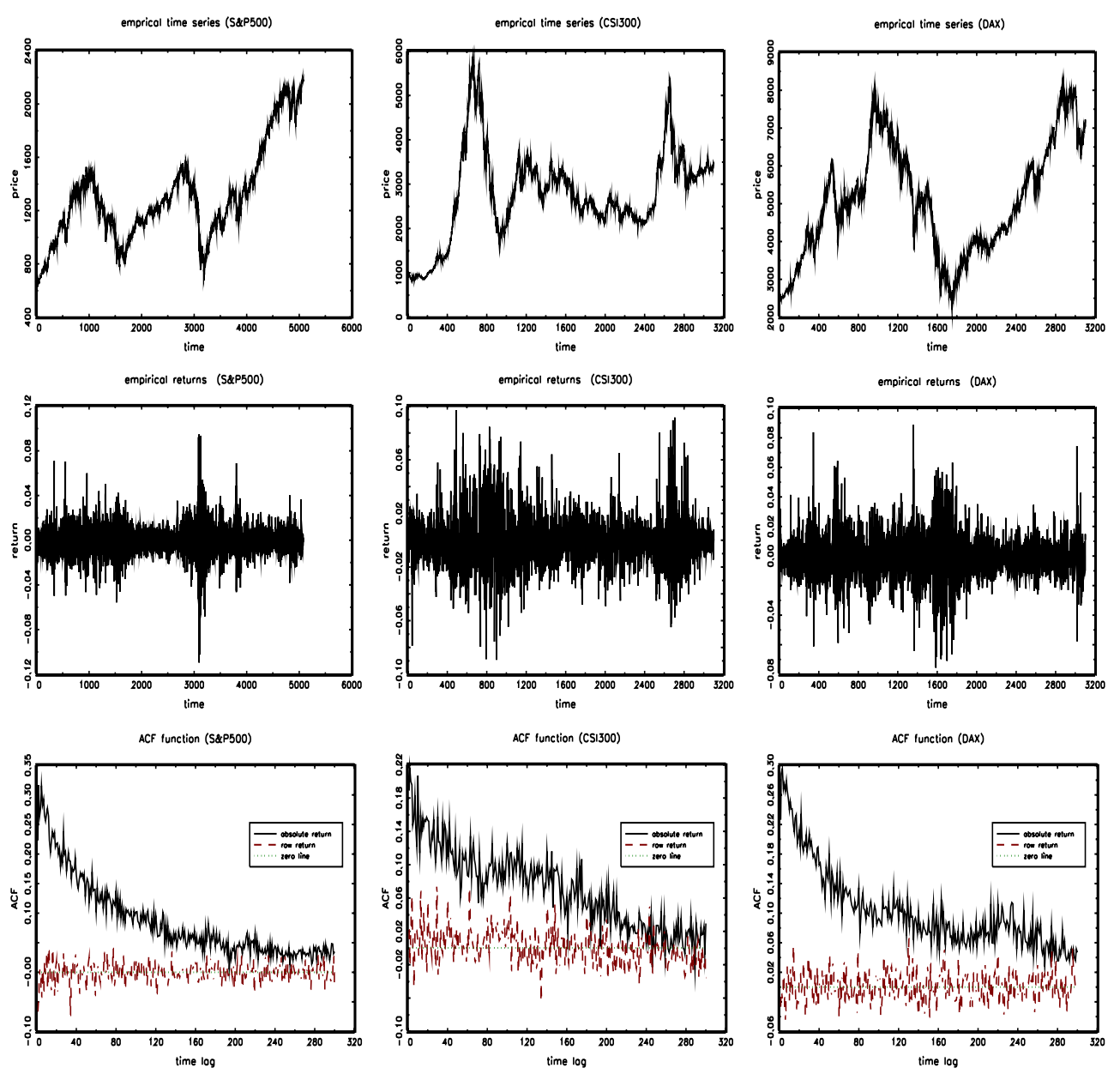

FiguRE 11. Empirical plots of stock markets indices.

truncation lag values, and more specifically, that they are monotonic decreasing. The right panel of Table 7 reports the Hurst exponents $H$ computed based on Lo's modified $R / S$ method. We observe the empirical values of $H$ are decreasing when $\tau$ increases. The results are consistent with the empirical plots, i.e., there exists apparently long memory in the return volatilities, and the strength of the long memory is diminishing with increasing time lags.

We then perform the modified rescaled range $(R / S)$ analysis with simulated data from our model (3.1) based on the parameter values given in Table 1. Table 8 presents mean and standard deviation of Lo's statistics for simulated 250 time series for different parameter values of $\left\{b, k, c, \beta, \gamma, F^{*}, \omega\right\}$ and for different truncation lags $\tau$ from 1 up to 250 . We find that the values are varying with different truncation lags and they are decreasing with increasing $\tau$, which show the similar pattern as reported for the empirical statistics. In addition, we observe that, given each individual parameter value of $\left\{b, c, \beta, F^{*}, \omega\right\}$, the test statistic values do not vary much for the same truncation lag $\tau$. However, for each individual parameter value 
of $\{k, \gamma\}$, we find that the Lo's $R / S$ statistics are decreasing with increasing the parameter values of $k$ from 0.1 to 0.9 , and $\gamma$ from 0.2 to 1 for a given time lag $\tau$.

Table 9 reports the number of rejections of the null hypothesis of short-range dependence at the $95 \%$ confidence levels. The rejection numbers for each single parameter value are decreasing as the truncation lag $\tau$ increases, and the proportion of rejections remains relatively high for even $\tau=250$. Analogously, there are not much variations for the number of rejections with different individual parameter values of $\left\{b, c, \beta, F^{*}, \omega\right\}$, but there are less frequent rejecting the null hypothesis of short-range dependence when the parameter values of $k$ and $\gamma$ increase for a given time lag $\tau$, which is consistent with the results found in Table 8.

The corresponding Hurst exponent $H$ estimates are given in Table 10. As similar behavior observed for the empirical Hurst exponent $H$ reported in Table 7 , the values of $H$ for the simulated data are decreasing when $\tau$ increases. Again we find the similar Hurst exponent $H$ values reported across different parameter values of $\left\{b, c, \beta, F^{*}, \omega\right\}$, but $H$ values decreases with increasing the parameter values of $k$ and $\gamma$.

Overall, our findings demonstrate that the model is able to mimic the long memory of return volatility which is pervasively found in financial time series. Our results also reveal that the measurement of the symmetry for the chartists' response to the price trend $(b)$, the confident level of the chartists $(c)$, the level of the fundamental price $\left(F^{*}\right)$, the coefficient of the retracement strategy $(\omega)$, and the performance sensitivity parameter $(\beta)$ have no impacts on the long memory of volatility, but only $k$ and $\gamma$, which measure the speed of the price adjustment of the market maker at the equilibrium of demand and supply, have the impact on the long memory of volatility.

TABle 7 . Lo's $R / S$ statistic for the empirical data.

\begin{tabular}{|c|ccc|ccc|}
\hline & \multicolumn{3}{|c|}{ Lo's statistic } & \multicolumn{3}{c|}{ Lo's Hurst exponent } \\
\hline & S\&P 500 & CSI 300 & DAX 30 & S\&P 500 & CSI 300 & DAX 30 \\
\hline \multirow{2}{*}{$\tau=1$} & 3.301 & 3.72 & 4.048 & 0.64 & 0.663 & 0.664 \\
$\tau=10$ & 2.469 & 3.022 & 3.08 & 0.606 & 0.638 & 0.632 \\
$\tau=50$ & 1.55 & 2.045 & 1.968 & 0.551 & 0.589 & 0.58 \\
$\tau=100$ & 1.253 & 1.657 & 1.606 & 0.526 & 0.563 & 0.556 \\
$\tau=150$ & 1.118 & 1.448 & 1.436 & 0.513 & 0.546 & 0.543 \\
$\tau=250$ & 0.988 & 1.236 & 1.256 & 0.499 & 0.526 & 0.527 \\
& & & & & & \\
\hline
\end{tabular}

Note: This table presents Lo's statistics and the Hurst exponent for the empirical data with different $\tau$. 
TABle 8. Lo's $R / S$ statistic for the simulated data.

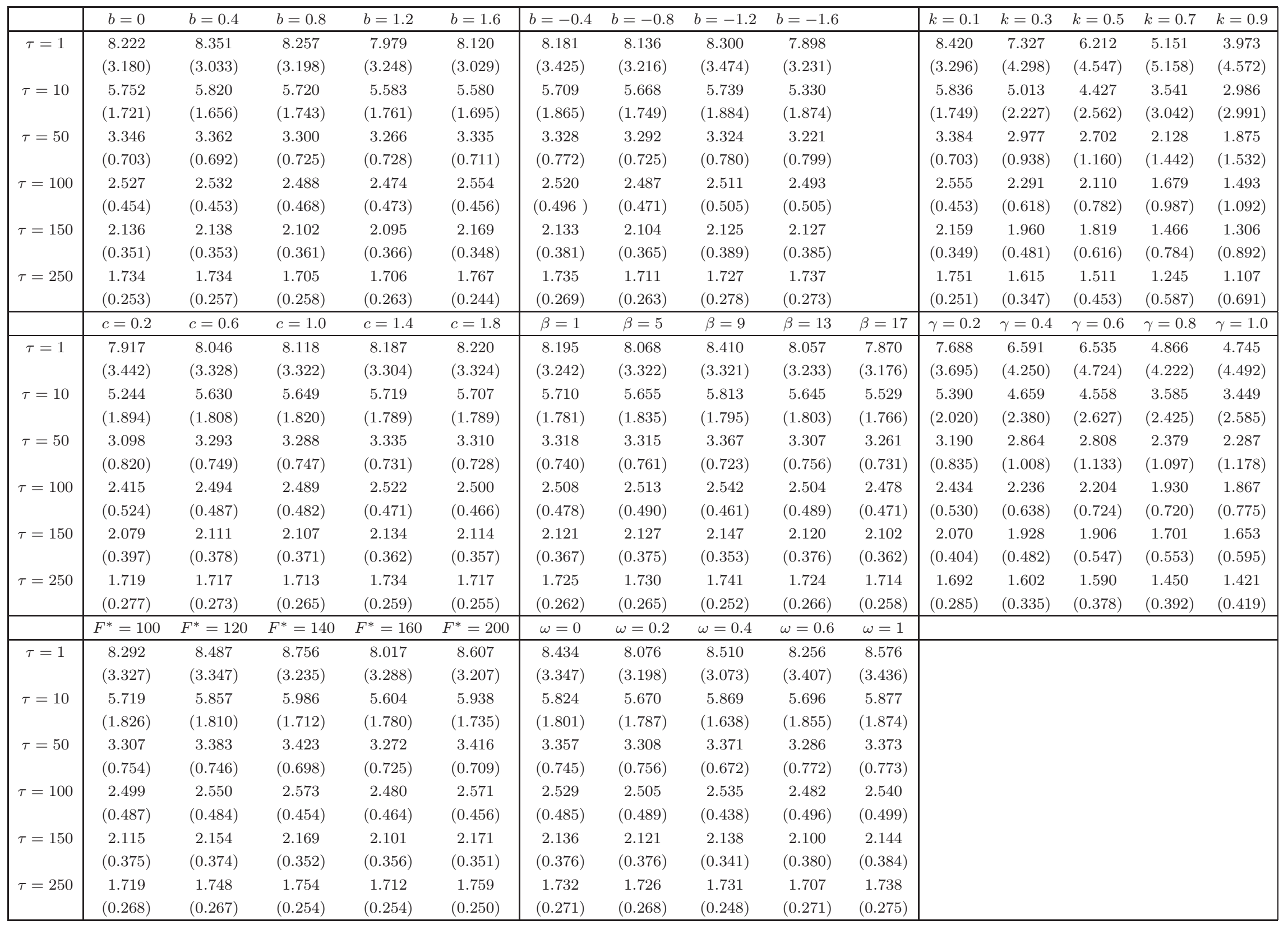

Note: This table presents the mean and standard deviation of Lo's statistics based on the corresponding 250 simulated time series with different $\tau$. Simulations are based on various parameters values of $\left\{b, k, c, \beta, \gamma, F^{*}, \omega\right\}$. 
TABLE 9. Lo's $R / S$ statistic test rejections.

\begin{tabular}{|c|c|c|c|c|c|c|c|c|c|c|c|c|c|c|c|}
\hline & $b=0$ & $b=0.4$ & $b=0.8$ & $b=1.2$ & $b=1.6$ & $b=-0.4$ & $b=-0.8$ & $b=-1.2$ & $b=-1.6$ & & $k=0.1$ & $k=0.3$ & $k=0.5$ & $k=0.7$ & $k=0.9$ \\
\hline$\tau=1$ & 245 & 249 & 248 & 246 & 249 & 244 & 249 & 245 & 247 & & 248 & 229 & 195 & 127 & 112 \\
\hline$\tau=10$ & 243 & 249 & 246 & 246 & 246 & 242 & 249 & 245 & 241 & & 248 & 229 & 197 & 128 & 115 \\
\hline$\tau=50$ & 243 & 241 & 238 & 239 & 242 & 238 & 245 & 238 & 230 & & 243 & 226 & 190 & 122 & 112 \\
\hline$\tau=100$ & 230 & 225 & 222 & 217 & 230 & 221 & 221 & 220 & 219 & & 233 & 195 & 172 & 102 & 97 \\
\hline$\tau=150$ & 197 & 198 & 189 & 187 & 205 & 193 & 176 & 192 & 194 & & 198 & 155 & 145 & 86 & 78 \\
\hline$\tau=250$ & 85 & 82 & 76 & 76 & 94 & 94 & 87 & 90 & 95 & & 91 & 63 & 50 & 50 & 48 \\
\hline & $c=0.2$ & $c=0.6$ & $c=1.0$ & $c=1.4$ & $c=1.8$ & $\beta=1$ & $\beta=5$ & $\beta=9$ & $\beta=13$ & $\beta=17$ & $\gamma=0.2$ & $\gamma=0.4$ & $\gamma=0.6$ & $\gamma=0.8$ & $\gamma=1.0$ \\
\hline$\tau=1$ & 245 & 247 & 245 & 246 & 246 & 246 & 244 & 247 & 243 & 247 & 238 & 209 & 192 & 178 & 155 \\
\hline$\tau=10$ & 243 & 246 & 245 & 246 & 243 & 242 & 244 & 247 & 242 & 245 & 237 & 207 & 190 & 176 & 153 \\
\hline$\tau=50$ & 234 & 237 & 238 & 242 & 237 & 239 & 239 & 242 & 239 & 240 & 229 & 197 & 184 & 159 & 141 \\
\hline$\tau=100$ & 203 & 217 & 222 & 229 & 224 & 226 & 220 & 226 & 216 & 222 & 215 & 179 & 170 & 129 & 121 \\
\hline$\tau=150$ & 177 & 194 & 187 & 194 & 192 & 198 & 194 & 204 & 194 & 189 & 180 & 150 & 147 & 100 & 91 \\
\hline$\tau=250$ & 94 & 82 & 83 & 89 & 78 & 82 & 89 & 83 & 86 & 87 & 75 & 69 & 65 & 41 & 39 \\
\hline & $F^{*}=100$ & $F^{*}=120$ & $F^{*}=140$ & $F^{*}=160$ & $F^{*}=200$ & $\omega=0$ & $\omega=0.2$ & $\omega=0.4$ & $\omega=0.6$ & $\omega=1$ & & & & & \\
\hline$\tau=1$ & 249 & 248 & 250 & 246 & 245 & 245 & 241 & 248 & 242 & 245 & & & & & \\
\hline$\tau=10$ & 249 & 247 & 249 & 244 & 245 & 245 & 241 & 248 & 242 & 245 & & & & & \\
\hline$\tau=100$ & 243 & 242 & 243 & 241 & 241 & 239 & 236 & 245 & 236 & 240 & & & & & \\
\hline$\tau=150$ & 219 & 224 & 227 & 225 & 229 & 226 & 224 & 226 & 223 & 224 & & & & & \\
\hline$\tau=250$ & 185 & 195 & 198 & 189 & 204 & 195 & 190 & 195 & 182 & 190 & & & & & \\
\hline$\tau=250$ & 87 & 100 & 92 & 78 & 99 & 94 & 85 & 85 & 79 & 98 & & & & & \\
\hline
\end{tabular}

Note: This table presents the number of Lo's $R / S$ statistic test rejections at the $5 \%$ level for the corresponding 250 simulated time series with different $\tau$ (the interval is $[0.809,1.862]$ as reported in Lo (1991) Table II). Simulations are based on various parameters values of $\left\{b, k, c, \beta, \gamma, F^{*}, \omega\right\}$. 
TABLE 10. Lo's $R / S$ Hurst exponents for the simulated data.

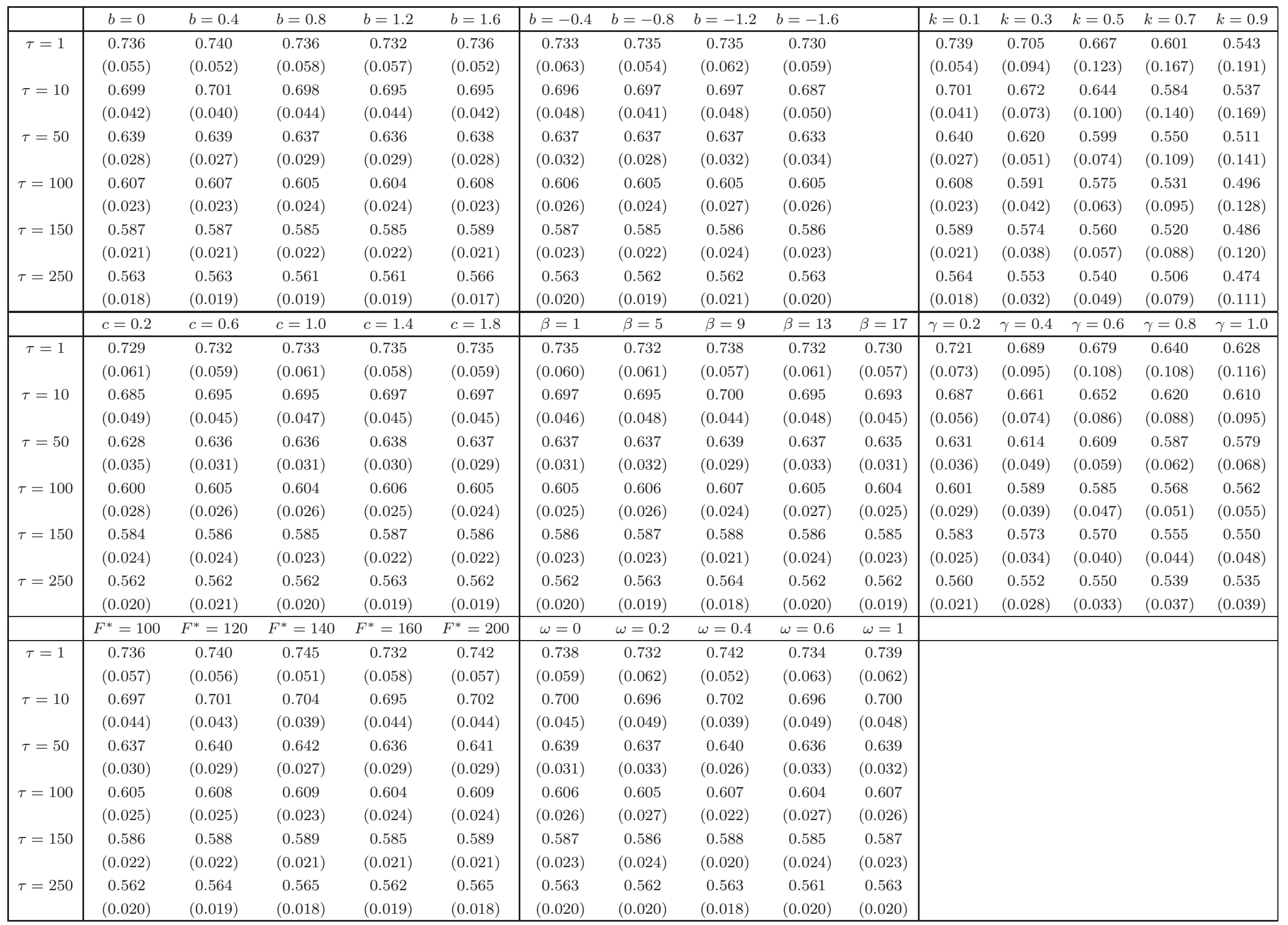

Note: This table presents the mean and standard deviation of Lo's Hurst exponent based on the corresponding 250 simulated time series with different $\tau$. Simulations are based on various parameters values of $\left\{b, k, c, \beta, \gamma, F^{*}, \omega\right\}$. 


\section{Conclusions}

We have introduced a parsimonious asset pricing model with boundedly rational agents who are specified as using simple heuristics in their decision making. Supporting the explanatory power of HAMs, the contributions of this paper are twofold. First, by analyzing the dynamical characteristics and stochastic simulations, we study the impact of the investors' behavior on the return volatility of a risky asset and provide additional support to the study of return volatility by using the theory of nonlinear dynamic systems, which describes the intrinsic characteristics of market volatility determined by the bounded rationality of investors. For example, we find that price limitation mechanism is essential to control the fluctuation amplitude of the market price, the level of the fundamentals affects the dynamics of the whole market, and the strategy and reaction intensity of the speculative behavior determine the stability of the market.

Second, in addition to the demonstrated ability of explaining the stylized facts of financial time series, we have examined the sources of the long memory, the phenomenon which has been ubiquitous in the financial markets, but very few studies on where the long memory comes from. We have applied the modified $R / S$ analysis to the simulated data according to our model, and found the speed of the price adjustment of the market maker can be a source of the volatility persistence.

Further study directions may include the validation of the boundedly rational agent model, which provides an avenue to a broader range of empirical applications, such as economic significance of difference trading strategies and volatility forecasting. 


\section{REFERENCES}

Alfarano, S., Lux, T. and Wagner, F. (2005), 'Estimation of agent-based models: The case of an asymmetric herding model', Computational Economics 26, 19-49.

Baak, S. (1999), 'Test for bounded rationality with a linear dynamics model distorted by heterogeneous expectations', Journal of Economic Dynamics and Control 23, 1517-1543.

Beja, A. and Goldman, M. (1980), 'On the dynamic behavior of prices in disequilibrium', Journal of Finance 35, 235-247.

Boswijk, H., Hommes, C. and Manzan, S. (2007), 'Behavioral heterogeneity in stock prices', Journal of Economic Dynamics and Control 31, 1938-1970.

Brock, W. and Hommes, C. (1997), 'A rational route to randomness', Econometrica 65, 1059-1095.

Brock, W. and Hommes, C. (1998), 'Heterogeneous beliefs and routes to chaos in a simple asset pricing model', Journal of Economic Dynamics and Control 22, 1235-1274.

Chavas, J. (2000), 'On the information and market dynamics: The case of the U.S. beef market', Journal of Economic Dynamics and Control 24, 833-853.

Chen, S.-H., Chang, C. and Du, Y. R. (2012), 'Agent-based economic models and econometrics', Knowledge Engineering Review 27(2), 187-219.

Chiarella, C. (1992), 'The dynamics of speculative behaviour', Annals of Operations Research 37, 101-123.

Chiarella, C., Dieci, R. and Gardini, L. (2002), 'Speculative behaviour and complex asset price dynamics', Journal of Economic Behavior and Organization 49, 173-197.

Chiarella, C., Dieci, R. and He, X. (2009), Heterogeneity, Market Mechanisms and Asset Price Dynamics, Elsevier, pp. 277-344. in Handbook of Financial Markets: Dynamics and Evolution, Eds. Hens, T. and K.R. Schenk-Hoppe.

Chiarella, C. and He, X. (2002), 'Heterogeneous beliefs, risk and learning in a simple asset pricing model', Computational Economics 19, 95-132.

Chiarella, C. and He, X. (2003), 'Heterogeneous beliefs, risk and learning in a simple asset pricing model with a market maker', Macroeconomic Dynamics 7, 503-536.

Chiarella, C., He, X. and Hommes, C. (2006), 'A dynamic analysis of moving average rules', Journal of Economic Dynamics and Control 30, 1729-1753.

Chiarella, C., He, X., Huang, W. and Zheng, H. (2012), 'Estimating behavioural heterogeneity under regime switching', Journal of Economic Behavior and Organization 83, 446-460.

Chiarella, C., He, X. and Zwinkels, R. (2014), 'Heterogeneous expectations in asset pricing: Empirical evidence from the S\&P 500', Journal of Economic Behavior and Organization 105, 1-16.

Chiarella, C., ter Ellen, S., He, X. and Wu, E. (2015), 'Fear or fundamentals? heterogeneous beliefs in the european sovereign cds market', Journal of Empirical Finance 32, 19-34.

Davies, R. B. and Harte, D. (1987), 'Tests for Hurst effect', Biometrika pp. 95-101.

Day, R. and Huang, W. (1990), 'Bulls, bears and market sheep', Journal of Economic Behavior and Organization 14, 299-329.

De Jong, E., Verschoor, W. and Zwinkels, R. (2010), 'Heterogeneity of agents and exchange rate dynamics: Evidence from the EMS', Journal of International Money and Finance 29(8), 16521669.

Dieci, R., Foroni, I., Gardini, L. and He, X. (2006), 'Market mood, adaptive beliefs and asset price dynamics', Chaos, Solitons and Fractals 29, 520-534.

Dieci, R. and He, X. (2018), 'Heterogeneous agent models in finance', Research Paper 389, Quantitative Finance Research Centre, University of Technology Sydney. 
Dieci, R. and Westerhoff, F. (2010), 'Heterogeneous speculators, endogenous fluctuations and interacting markets: A model of stock prices and exchange rates', Journal of Economic Dynamics and Control 34, 743-764.

Dieci, R. and Westerhoff, F. (2016), 'Heterogeneous expectations, boom-bust housing cycles, and supply conditions: a nonlinear economic dynamics approach', Journal of Economic Dynamics and Control 71, 21-44.

Franke, R. (2009), 'Applying the method of simulated moments to estimate a small agent-based asset pricing model', Journal of Empirical Finance 16, 804-815.

Franke, R. and Westerhoff, F. (2011), 'Estimation of a structural stochastic volatility model of asset pricing', Computational Economics 38, 53-83.

Franke, R. and Westerhoff, F. (2012), 'Structural stochastic volatility in asset pricing dynamics: Estimation and model contest', Journal of Economic Dynamics and Control 36, 1193-1211.

Frijns, B., Lehnert, T. and Zwinkels, R. (2010), 'Behavioral heterogeneity in the option market', Journal of Economic Dynamics and Control 34, 2273-2287.

Gaunersdorfer, A. and Hommes, C. (2007), A Nonlinear Structural Model for Volatility Clustering, Springer, Berlin/Heidelberge, pp. 265-288. in Long Memory in Economics, Eds. Teyssiere, G. and A. Kirman.

Gaunersdorfer, A., Hommes, C. and Wagener, F. (2008), 'Bifurcation routes to volatility clustering under evolutionary learning', Journal of Economic Behavior and Organization 67, 27-47.

Gilli, M. and Winker, P. (2003), 'A global optimization heuristic for estimating agent-based model', Computational Statistics and Data Analysis 42, 299-312.

Goldbaum, D. and Mizrach, B. (2008), 'Estimating the intensity of choice in a dynamic mutual fund allocation decision', Journal of Economic Dynamics and Control 32, 3866-3876.

He, X. (2014), Recent developments in asset pricing with heterogeneous beliefs and adaptive behavior of financial markets, Springer, pp. 3-34. in Global Analysis of Dynamical Models in Economics and Finance, Eds. G.I Bischi, C. Chiarella, and I. Sushko.

He, X., Li, K. and Wang, C. (2016), 'Volatility clustering: A nonlinear theoretical approach', Journal of Economic Behavior and Organization 130, 274-297.

He, X. and Li, Y. (2007), 'Power law behaviour, heterogeneity, and trend chasing', Journal of Economic Dynamics and Control 31, 3396-3426.

He, X. and Li, Y. (2008), 'Heterogeneity, convergence and autocorrelations', Quantitative Finance 8, 58-79.

He, X. and Li, Y. (2015), 'Testing of a market fraction model and power-law behaviour in the DAX 30', Journal of Empirical Finance 30, 1-17.

He, X. and Li, Y. (2017), 'The apadtiveness in stock markets: testing the stylized facts in the DAX 30', Journal of Evolutionary Economics 27, 1071-1094.

Hommes, C. (2001), 'Financial markets as nonlinear adaptive evolutionary systems', Quantitative Finance 1, 149-167.

Hommes, C. (2006), Heterogeneous Agent Models in Economics and Finance, Vol. 2 of Handbook of Computational Economics, North-Holland, pp. 1109-1186. in Agent-based Computational Economics, Eds. Tesfatsion, L. and K.L. Judd.

Hurst, E. (1951), 'Long term storage capacity of reservoirs', Transactions on American Society of Civil Engineering 116, 770-808. 
LeBaron, B. (2006), Agent-based Computational Finance, Vol. 2 of Handbook of Computational Economics, North-Holland, pp. 1187-1233. in Agent-based Computational Economics, Eds. Tesfatsion, L. and K.L. Judd.

Li, Y., Donkers, B. and Melenberg, B. (2010), 'Econometric analysis of microscopic simulation models', Quantitative Finance 10, 1187-1201.

Lillo, F. and Farmer, J. (2004), 'The long memory of the efficient market', Studies in Nonlinear Dynamics and Econometrics 8(3), Article 1.

Lo, A. (1991), 'Long-term memory in stock market prices', Econometrica 59, 1279-1313.

Lux, T. (1995), 'Herd behaviour, bubbles and crashes', Economic Journal 105, 881-896.

Lux, T. (2009a), 'Rational forecasts or social opinion dynamics? identification of interaction effects in a business climate survey', Journal of Economic Behavior and Organization 32, 638-655.

Lux, T. (2009b), Stochastic Behavioural Asset Pricing and Stylized Facts, Elsevier, pp. 161-215. in Handbook of Financial Markets: Dynamics and Evolution, Eds. Hens, T. and K.R. SchenkHoppe.

Lux, T. (2012), 'Estimation of an agent-based model of investor sentiment formation in financial markets', Journal of Economic Behavior and Organization 36, 1284-1302.

Lux, T. and Alfarano, S. (2016), 'Financial power laws: Empirical evidence, models, and mechanisms', Chaos, Solitons 83 Fractals 88, 3-18.

Mandelbrot, B. and Taqqu, M. (1979), 'Robust R/S analysis of long-run serial correlation', Bulletin of the International Statistical Institute 48, 69-104.

Mandelbrot, B. and Wallis, J. (1969), 'Robustness of the rescaled range R/S in the measurement of non noncyclic long-run statistical dependence', Water Resources Research 5, 967-988.

Manzan, S. and Westerhoff, F. (2005), 'Representativeness of news and exchange rate dynamics', Journal of Economic Dynamics and Control 29, 677-689.

Schmitt, N. and Westerhoff, F. (2014), 'Speculative behavior and the dynamics of interacting stock markets', Journal of Economic Dynamics and Control 45, 262-288.

ter Ellen, S., Verschoor, W. and Zwinkels, R. (2013), 'Dynamic expectation formation in the foreign exchange market', Journal of International Money and Finance 37, 75-97.

ter Ellen, S. and Zwinkels, R. (2010), 'Oil price dynamics: a behavioral finance approach with heterogeneous agents', Energy Economics 32, 1427-1434.

Westerhoff, F. and Franke, R. (2014), OUP Handbook on Computational Economics and Finance, Oxford University Press, Oxford, chapter Agent-based models for economic policy design: two illustrative examples.

Westerhoff, F. and Reitz, S. (2003), 'Nonlinearities and cyclical behavior: The role of chartists and fundamentalists', Studies in Nonlinear Dynamics and Econometrics 7(4), article no. 3.

Zheng, M., Wang, D. and He, X. (2009), 'Asymmetry of technical analysis and market price volatility', China Finance Review 3(2), 61-89.

Zheng, M., Wang, H., Wang, C. and Wang, S. (2017), 'Speculative behavior in a housing market: Boom and bust', Economic Modelling 61, 50-64. 